\title{
Laser-modified PLGA for implants: in vitro degradation and mechanical properties
}

\author{
MAGDALENA KOBIELARZ ${ }^{1 *}$, MAGDALENA TOMANIK ${ }^{1}$, \\ KATARZYNA MROCZKOWSKA ${ }^{2}$, KONRAD SZUSTAKIEWICZ ${ }^{3}$, MAGDALENA ORYSZCZAK ${ }^{4}$, \\ ANNA MAZUR ${ }^{4}$, ARKADIUSZ ANTOŃCZAK $^{2}$, JAROSŁAW FILIPIAK $^{1}$ \\ ${ }^{1}$ Department of Mechanics, Materials and Biomedical Engineering, Faculty of Mechanical Engineering, \\ Wrocław University of Science and Technology, Wrocław, Poland. \\ ${ }^{2}$ Laser and Fiber Electronics Group, Faculty of Electronics, \\ Wrocław University of Science and Technology, Wrocław, Poland. \\ ${ }^{3}$ Department of Polymer Engineering and Technology, Faculty of Chemistry, \\ Wroclaw University of Science and Technology, Wrocław, Poland. \\ ${ }^{4}$ Scientific Circle of Biomechanics, Faculty of Mechanical Engineering, \\ Wrocław University of Science and Technology, Wrocław, Poland.
}

Purpose: Irradiations of poly(lactic-co-glycolic acid) surface by $\mathrm{CO}_{2}$ laser lead to alterations of physicochemical properties of the copolymer. Effects of PLGA irradiations depend on the process parameters determining different cases of surface modification. Hence the main goal of presented studies was to define the influence of $\mathrm{CO}_{2}$ laser irradiation with different process parameters, inducing three cases of surface modification, on mechanical properties and topography of PLGA during degradation in the aqueous environment. Methods: Hydrolytic degradation of untreated and treated by $\mathrm{CO}_{2}$ laser thin specimens of PLGA was performed in distilled (demineralized) water. Mechanical properties of PLGA specimens before and during incubation were conducted in accordance with the PN-EN ISO 527-3:1998 standard. The pH of incubation solutions, topographies, masses and geometrical dimensions of specimens were controlled during the process. Results: During the hydrolytic degradation, gradual changes in failure mode were observed from ductile failure characteristic for untreated PLGA to brittle failure of incubated PLGA regardless of the case of induced modification. Tensile strength decreased with degradation time regardless of the case of surface modification with insignificant fluctuation Young's moduli at the level of means. The $\mathrm{pH}$ of solutions for each case decreased and topography od specimens become smoother with incubation time. Conclusions: PLGA surface modification by $\mathrm{CO}_{2}$ laser below the ablation threshold (P1) and at the ablation threshold (P2) led to surface functionalization, however, irradiation above the ablation threshold (P3) caused marked degradation of PLGA and accelerated specimens disintegration during incubation in the aquatic environment.

Key words: biodegradable polymers, poly(lactic-co-glycolic acid), implants, laser surface modifications, mechanical properties

\section{Introduction}

In the first two decades of the 21 st century, there has been a growing interest in, and use of, polymer biomaterials in medicine. Particularly noteworthy are bioresorbable polymers that are used to produce various types of implants and scaffolds for tissue engineering, including poly(glycolic acid) (PGA), poly(lactic acid) (PLA) as well as poly(lactic-co-glycolic acid) (PLGA) copolymers based on the above polymers. The main advantage of these synthetic biocompatible polymers is their degradation as a result of hydrolysis in aqueous solution of the ester backbone to acid monomers, which are then metabolised or eliminated by the body. During the initial treatment period, an implant made

* Corresponding author: Magdalena Kobielarz, Department of Mechanics, Materials and Biomedical Engineering, Faculty of Mechanical Engineering, Wrocław University of Science and Technology, ul. Łukasiewicza 7/9, 50-371 Wrocław, Poland, e-mail: magdalena.kobielarz@pwr.edu.pl

Received: December 14th, 2019

Accepted for publication: January 18th, 2020 
of bioresorbable polymers is the main element responsible for the transfer of loads and restoration of the damaged tissue structure. As the treatment progresses, the role of the implant gradually decreases due to its degradation and the regenerating tissue structure regains the ability to perform its function. Consequently, the mechanical properties of the implant systematically decrease while ensuring gradual loading of the regenerating tissue structure. This is consistent with the expected biomechanical matching of the mechanical properties of the implant and tissue [26].

In practice, many distinct polymers are used, having different functional parameters, such as the time of reduction of mechanical properties, degradation time, and physicochemical properties. This study focuses on PLGA, which is a copolymer of lactic acid and glycolic acid, whose properties depend on the percentages of individual components [3], [10]. Its tensile strength can range from 35 to $61 \mathrm{MPa}$, Young's modulus from 0.67 to $4.5 \mathrm{GPa}$, and tensile strain from 1.9 to $10 \%$ [7]. Depending on the PLA/PGA ratio, it is possible to control the degree of crystallinity, hydrophilicity, and glass-transition temperature as well as mechanical properties of the material, but also its degradation time and release rate of medicinal substances incorporated into the polymer [17], [19]. As with other thermoplastic polymers, PLGA has features that can be troublesome in bio-medicinal applications. This applies to the very low roughness and low wettability of the surface. These are important parameters determining the interaction between the cells and the implant surface. A higher adhesion of cells to the implant surface is determined both by varied topography characterized by a higher roughness and by hydrophilicity of the surface. The appropriate roughness and hydrophilicity of the surface provide suitable conditions for colonization by cells thanks to better adhesion and, consequently, better integration of the forming tissue with the implant. The issue of surface modification of bioresorbable polymers is related to the possibility of functionalisation of the surfaces of various types of implants in order to increase their ability to interact with the tissue.

As in other polymers, physicochemical and mechanical properties of PLGA can be changed by volume and/or surface modifications [7], [25]. The effects of volumetric modifications of polymers are easier to control because the structure changes throughout the volume of the material [4], [29]. By contrast, in the case of surface modifications, the use of such techniques as coating, low-temperature plasma treatment, and laser radiation [15], [18] leads to changes mainly in the surface layer of the treated material. One of the most promising techniques for surface modification of polymers is laser processing. Modification of the polymer surface with a laser beam is the result of two basic mechanisms: photochemical and photothermal [2], [4], [20], [25]. It is well known that heat acting on polylactone surfaces causes various processes leading to degradation of the material, including random chain breaking, depolymerization, oxidation degradation, intramolecular and intermolecular transesterifications, and hydrolysis [5], [28]. Photochemical processes initiate the breaking of chemical bonds by high-energy photons that activate a number of reactions such as crosslinking, chain scission, and formation of radicals [20]. The coexistence of the two mechanisms and the thermal effects accompanying the laser irradiation process both in the zone planned for modification and in the heat-affected zone (HAZ) make the effects of laser surface modifications difficult to estimate based on the parameters of the process itself [2], [13], [22], [25]. It should be emphasized that, in addition to microstructural modification, laser radiation may also affect morphology of the surface, determining changes to its roughness and wettability, which translates into surface cytocompatibility [22], [30]. To the best of our knowledge, there are few studies describing the effects of laser treatment on the mechanical properties of biodegradable polymers for medical applications. Moreover, the literature lacks description of the impact of hydrolytic degradation, so characteristic for biodegradable polymers, on the strength parameters.

In the present study, research was undertaken to assess the effects of surface modifications of thin PLGA films with a $\mathrm{CO}_{2}$ laser beam on the degradation rate and mechanical properties of the material. The research focused on modification of thin films, because PLGA is used for the production of delicate implants, such as scaffolds for tissue engineering or bioresorbable vascular stents, where thickness of structural elements ranges from 200 to 400 micrometres.

\section{Materials and methods}

\subsection{Materials}

The research material was PLGA in the percentage ratio of lactide to glycolide of 85:15 (Evonik, LG857s). The PLGA foils with an average thickness of $350 \mu \mathrm{m}$ were produced by compression moulding of granules melted at a temperature of $200{ }^{\circ} \mathrm{C}$. Ultimately, an amorphous material was obtained, whose degree of 
crystallinity was $X c \approx 2-3 \%$ [14]. From the obtained films, standardized dumbbell specimens type $5 \mathrm{~A}$ were cut out with a blanking die. A total of 80 specimens were cut out, of which 60 specimens were subjected to surface modifications using a $\mathrm{CO}_{2}$ laser with three different parameters of the irradiation process (Table 1), while the remaining 20 specimens constituted the reference group (REF).

Table 1. Process parameters for surface modifications of PLGA specimens with a $\mathrm{CO}_{2}$ laser

\begin{tabular}{|l|c|c|c|}
\hline \multirow{2}{*}{$\begin{array}{c}\text { Cases of surface } \\
\text { modification }\end{array}$} & \multicolumn{3}{|c|}{$\begin{array}{c}\text { Parameters of the laser } \\
\text { modification process }\end{array}$} \\
\cline { 2 - 4 } & $\begin{array}{c}\text { Pulse } \\
\text { numbers }\end{array}$ & $\begin{array}{c}\text { Pulse energy } \\
{[\mathrm{mJ}]}\end{array}$ & $\begin{array}{c}\text { Accumulated } \\
\text { fluencies } \\
{\left[\mathrm{mJ} / \mathrm{cm}^{2}\right]}\end{array}$ \\
\hline $\begin{array}{l}\text { Reference material } \\
\text { (REF) }\end{array}$ & - & - & - \\
\hline $\begin{array}{l}\text { Below the ablation } \\
\text { threshold (P1) }\end{array}$ & 5 & 4.72 & 24 \\
\hline $\begin{array}{l}\text { At the ablation } \\
\text { threshold (P2) }\end{array}$ & 6 & 7.49 & 48 \\
\hline $\begin{array}{l}\text { Above the ablation } \\
\text { threshold (P3) }\end{array}$ & 6 & 11.73 & 71 \\
\hline
\end{tabular}

The surfaces of PLGA specimens were modified with an air-cooled RF-excited pulsed $\mathrm{CO}_{2}$ laser $(10.6 \pm 0.03 \mu \mathrm{m})-$ Series $48-2$ Synrad, Inc. with the maximum average power of $25 \mathrm{~W}$ and pulse duration ranging from several dozen to several hundred $\mu \mathrm{s}$ (depending on the power and the pulse repetition rate). The beam with the mode purity $\mathrm{TEM}_{00}$ at $95 \%$ (beam quality factor $M^{2}<1.2$ ) was focused on the surface of material using a $38.1 \mathrm{~mm}\left(1.5^{\prime \prime}\right)$ focal lens. The irradiation parameters were selected in such a way (based on our previous work) as to obtain three cases of surface modification [2] as follows: (i) without ablation of the material, which allows products of the photothermal process to accumulate on the surface. In this case the penetration depth of the $\mathrm{CO}_{2}$ laser was smaller than the thickness of the sample, thus the modifications mainly affected the surface layer of the polymer (P1); (ii) with ablation but without significant loading of the material with residual heat of the proc- ess (reduced thermal effects) (P2); and (iii) with ablation of used material approximately to half of its thickness, for this fluence the polymer was modified in all its volume (P3). The following accumulated $\mathrm{CO}_{2}$ laser fluences were used for the successive cases of material surface modifications: (i) $24 \mathrm{~J} / \mathrm{cm}^{2}-$ below the ablation threshold (case P1), (ii) $48 \mathrm{~J} / \mathrm{cm}^{2}-$ at the ablation threshold (case P2), and (iii) $71 \mathrm{~J} / \mathrm{cm}^{2}$ - above the ablation threshold (case P3) (Table 1).

\subsection{Hydrolytic degradation}

All the specimens were subjected to the hydrolytic degradation process in accordance with the degradation calendar (Table 2). At each stage of the degradation, 5 specimens of the reference material (REF) and 5 specimens of each type of surface modification (P1, P2, and P3) were incubated. The process of hydrolytic degradation was carried out in test tubes with distilled (demineralized) water with the volume of $15 \mathrm{ml}$ at a temperature of $37{ }^{\circ} \mathrm{C}$ in a temperature chamber (Pol-Eko, CLN 115). The incubation solution was changed every 2 weeks.

\subsection{Mechanical properties}

Testing of the mechanical properties of the PLGA reference specimens (REF), i.e., the specimens not irradiated with a $\mathrm{CO}_{2}$ laser, and of the three cases of surface modification took place at individual stages of the process of hydrolytic degradation in accordance with the degradation calendar (Table 2). The tests were carried out on MTS 858 Mini Bionix testing machine fitted with hydraulic grips ensuring constant clamping force on the tested specimen during the entire stretching process. Stretching speed was $4 \mathrm{~mm} / \mathrm{min}$ [12]. During testing, tensile force and specimen elongation were recorded. The stress values were determined as the ratio of the force at a given moment of the test to the initial crosssectional area of the specimen. The strain value was

Table 2. Degradation calendar of PLGA specimens

\begin{tabular}{|l|c|c|c|c|c|c|}
\hline \multirow{2}{*}{ Cases of surface modification } & \multicolumn{5}{|c|}{ Degradation time [weeks] } & \multirow{2}{*}{$\begin{array}{c}\text { Specimens } \\
\text { labeling }\end{array}$} \\
\cline { 2 - 7 } & Before & I stage & II stage & III stage & IV stage & PLGA_REF \\
\hline Reference material (REF) & 0 & 4 & - & 8 & 12 & PLG_ \\
\hline Below the ablation threshold (P1) & 0 & 4 & - & 8 & 12 & PLGA_P1 \\
\hline At the ablation threshold (P2) & 0 & 3 & 6 & 9 & 12 & PLGA_P2 \\
\hline Above the ablation threshold (P3) & 0 & 3 & 6 & 9 & 12 & PLGA_P3 \\
\hline
\end{tabular}


determined as the ratio of the increase in the specimen length to its initial length, with the increase in the length of the specimen being determined on the basis of the change in the distance between the grips. The obtained results were used to plot the stress-strain characteristics, which allowed to determine Young's modulus $(E)$ and tensile strength $\left(R_{m}\right)$. Young's modulus was determined based on the linear segment of the curve $\sigma=f(\varepsilon)$ in the strain range of $0.005-0.025$ in accordance with PN-EN ISO 527-3:1998 [12].

\subsection{Surface topography}

At individual stages of hydrolytic degradation, microscopic analyses of the surface topography of the specimens were carried out using a video microscope (Micro-Combi Tester, CSM Instruments). The assessment of the topography made it possible to observe the degradation progress on the surface of the specimens.

\subsection{Mass and geometric dimensions of the specimens}

The geometric dimensions of the specimens were measured before the start of incubation (baseline measurement), and then after each completed stage of the degradation (in accordance with the degradation calendar). Thickness was measured with Coolant Proof IP65 micrometre, while width was determined with Zeiss Discovery V20 microscope. The specimens were weighed before the start of incubation, after each completed stage of the degradation, and after 48 hours of air drying at room temperature, which enabled an assessment of the degree of water absorption by the material. Mass measurements were carried out using Radwag ${ }^{\circledR}$ Ps $1000 / C / 2$ analytical balance.

\section{6. $\mathrm{pH}$ of the degradation environment}

The dynamics of the release of degradation products were examined by assessing the $\mathrm{pH}$ value of the incubation solution. The $\mathrm{pH}$ of the incubation solution was measured every 2 weeks. The measurements were performed with Elmetron CX-505 multifunction meter equipped with EPS-1 electrode for measuring $\mathrm{pH}$. In order to identify the nature and direction of changes in the $\mathrm{pH}$ of the incubation solution, a linear function $f(x)=a x \pm b$ approximating the determined mean $\mathrm{pH}$ values was determined in the successive weeks of the degradation of the PLGA specimens.

\subsection{Statistical analysis}

The impact of hydrolytic degradation on changes in the mechanical parameters of both reference and laser-modified PLGA specimens was analysed using the Statistica 13.1 software package. The significance of the differences was analysed using the Friedman and Kruskal-Wallis tests to evaluate the effects of the degradation time and modification case, respectively, on the mechanical properties of PLGA. Statistical significance was assumed as $\alpha=0.05$. All the results were presented as mean and standard deviations $(\bar{X} \pm S D)$.

\section{Results}

\subsection{Mechanical properties}

A uniaxial tensile test of the PLGA specimens, including reference specimens and specimens modified by a $\mathrm{CO}_{2}$ laser with different process parameters, was used to determine the stress-train characteristics for all stages of hydrolytic degradation. PLGA reference specimens before degradation ( 0 weeks) underwent ductile failure, whereas the mode of failure of PLGA specimens treated by a $\mathrm{CO}_{2}$ laser and not subjected to hydrolytic degradation ( 0 weeks) became more brittle as fluence increased, which was described in detail by Kobielarz et al. [14]. Similar changes occurred in the process of the degradation, where in successive stages the mode of failure changed with the degradation time towards brittle failure (Fig. 1). As the degradation time increased, the range of plastic deformations (plastic region) and strain softening became smaller, regardless of the fluence used for surface modification.

The mechanical properties, i.e., Young's modulus (E) and tensile strength $\left(R_{m}\right)$, of PLGA reference specimens and PLGA specimens irradiated by a $\mathrm{CO}_{2}$ laser with different fluences (P1, P2, and P3) at individual stages of hydrolytic degradation were determined in accordance with the PN-EN ISO 527-3:1998 standard [12]. The analysis assessed changes in Young's modulus and tensile strength occurring with the progress of the degradation (in successive weeks/stages) separately for reference specimens and for individual cases of modification of PLGA surfaces (Fig. 2). In the next stages of the degradation, the analysis concerned the effects of laser surface modification on the mechanical parameters in relation to the unmodified reference material (REF) were performed (Fig. 3). 

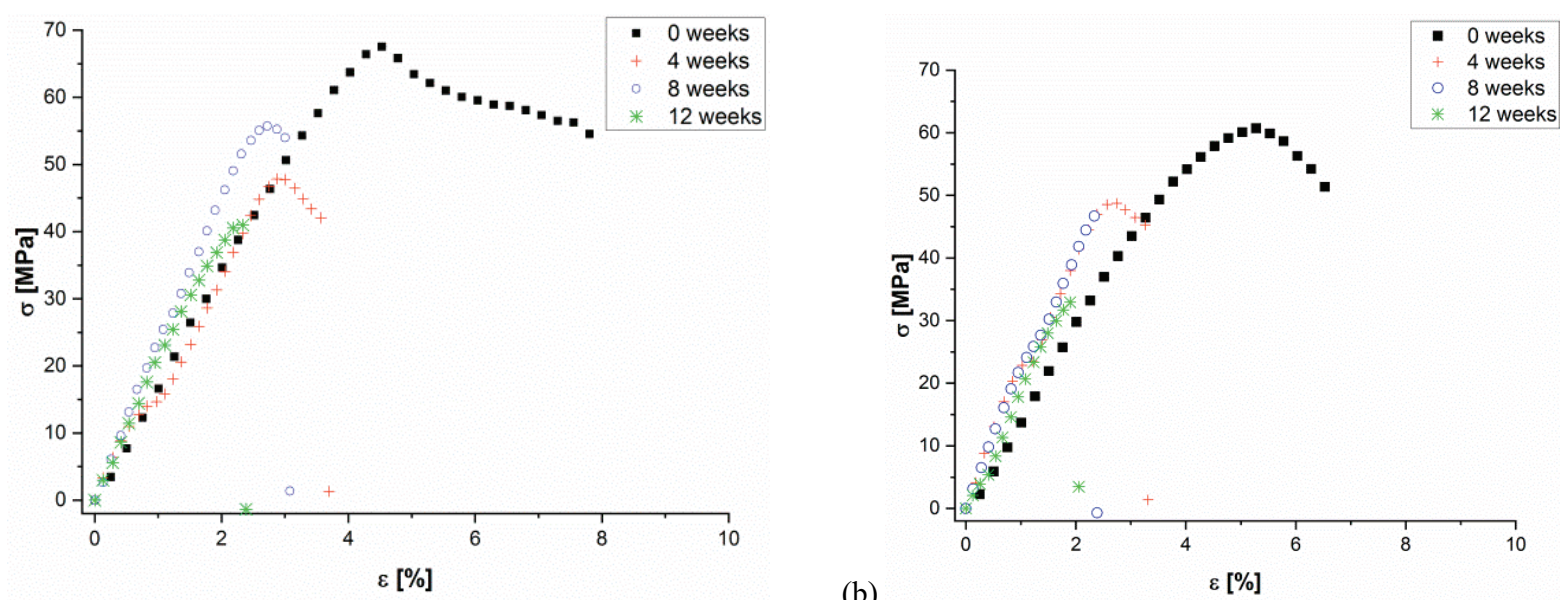

(a)

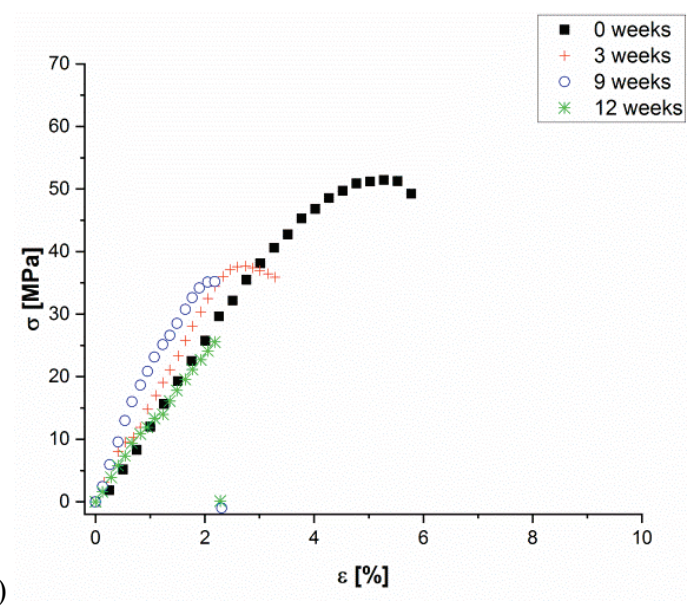

(b)

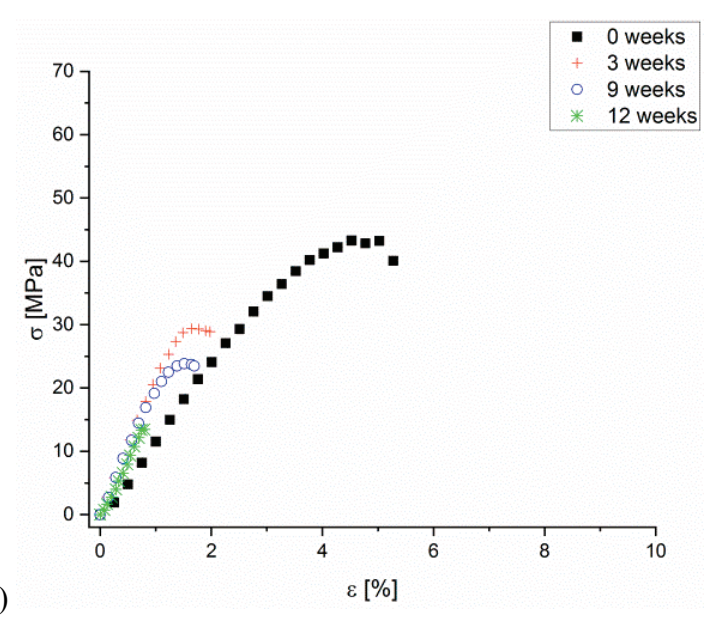

Fig. 1. Examples of stress-strain curves after different PLGA degradation times:

(a) reference specimens, (b) specimens modified with the P1 fluence,

(c) specimens modified with the P2 fluence, and (d) specimens modified with the P3 fluence

Tensile strength in both reference (Fig. 2a) and laser-irradiated (Figs. 2c, 2e, and 2g) PLGA specimens decreased with the degradation time regardless of the case of induced modification. After the first stage of the degradation (third or fourth week), the $R_{m}$ value fell rapidly and then the dynamics of changes decreased. After 12 weeks of the degradation, tensile strength again dropped sharply for PLGA_REF and PLGA_P1. The Young's modulus values for PLGA reference specimens were not statistically significantly different in individual degradation periods (Fig. 2b). There was, however, a slight reduction in the mean value of the modulus after the first stage of incubation (4 weeks), followed by a gradual increase in the value of Young's modulus. On the other hand, an upward trend in the values of Young's modulus was observed in the early stages of the degradation in the case of PLGA specimens irradiated with a laser, regardless of the fluence used (Figs. 2d, 2f, and 2h). A breakdown or reversal of this trend occurred earlier at higher applied fluence values, i.e., after degradation lasting 6 weeks in the case of PLGA_P3, 9 weeks in the case of PLGA_P2, or 12 weeks in the case of PLGA_P1.

Before the degradation ( 0 weeks), tensile strength and Young's modulus (Figs. 3a and 3b) decreased with increasing value of the fluence used for irradiation. In successive stages of the degradation, tensile strength (Figs. 3c, 3e, 3g, and 3i) also gradually decreased with increasing fluence values, with the means obtained for PLGA_REF and PLGA_P1 being similar. A clear decline in the $R_{m}$ value was only noted for the successive case of modification (PLGA_P2) and the next reduction of the $R_{m}$ values was observed for PLGA_P3. Young's modulus at stages I (Fig. 3d) and II (Fig. 3f) of the degradation did not significantly change. Only at stage III (Fig. 3h) PLGA_P2 and PLGA_P3 specimens had an evidently lower Young's moduli than PLGA_REF and PLGA_P1 specimens. After a 12-week incubation (Fig. 3j), there was a clear decrease in the value of $E$ correlated with an increase in the value of fluence. PLGA_REF after a 12-week degradation showed a large diversity of the recorded 
Tensile strength

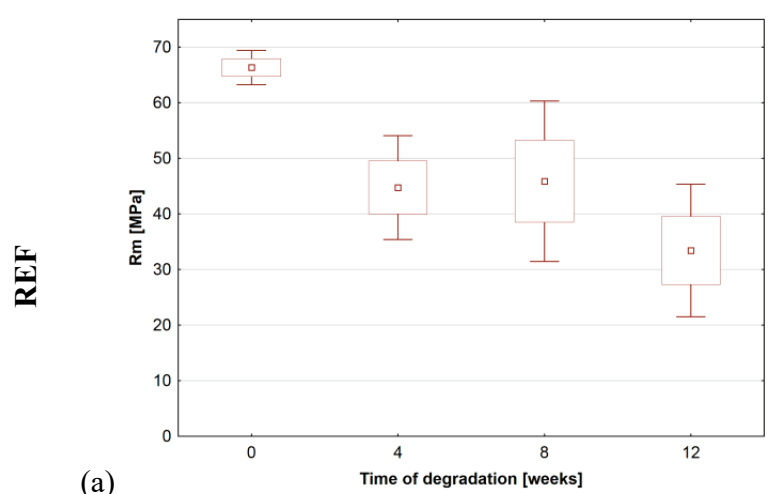

(a)

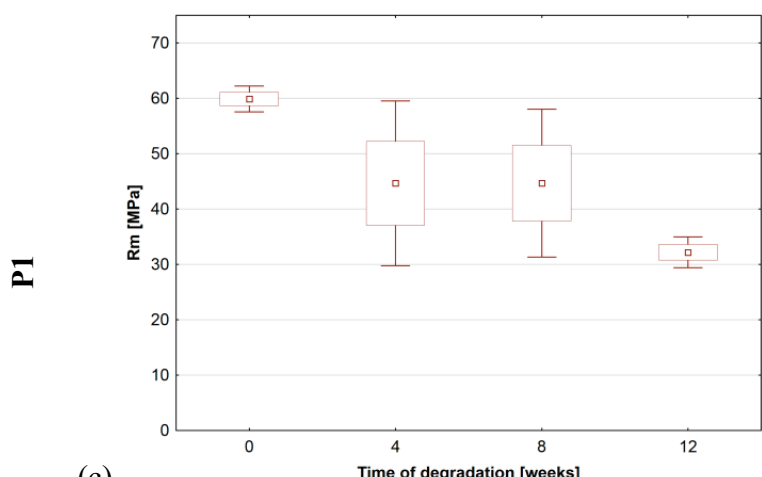

(c)
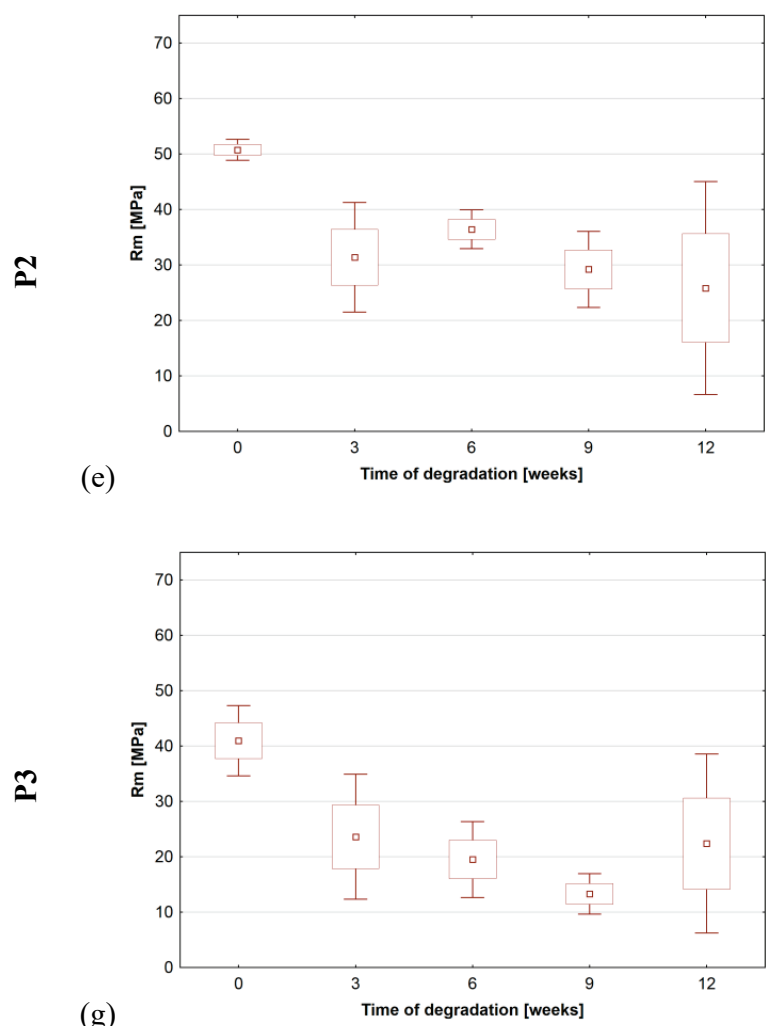

Young's modulus

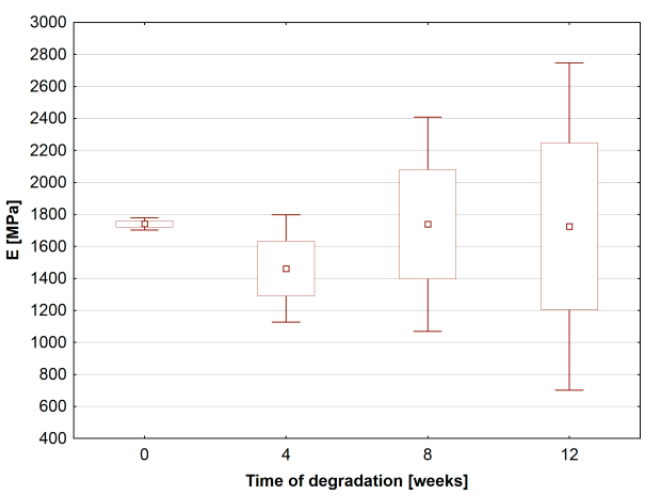

(b)

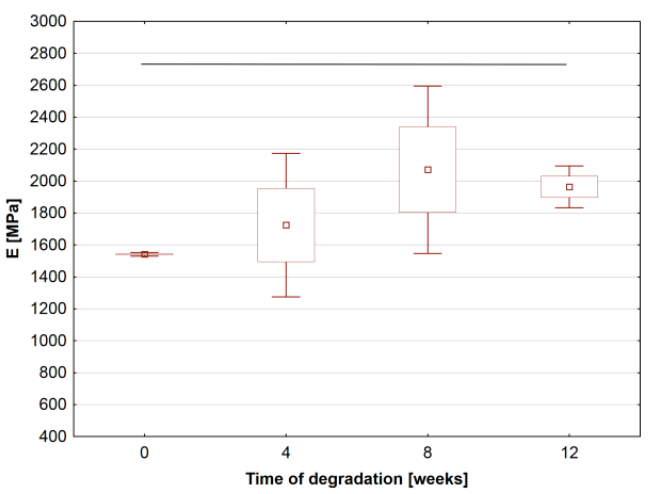

(d)
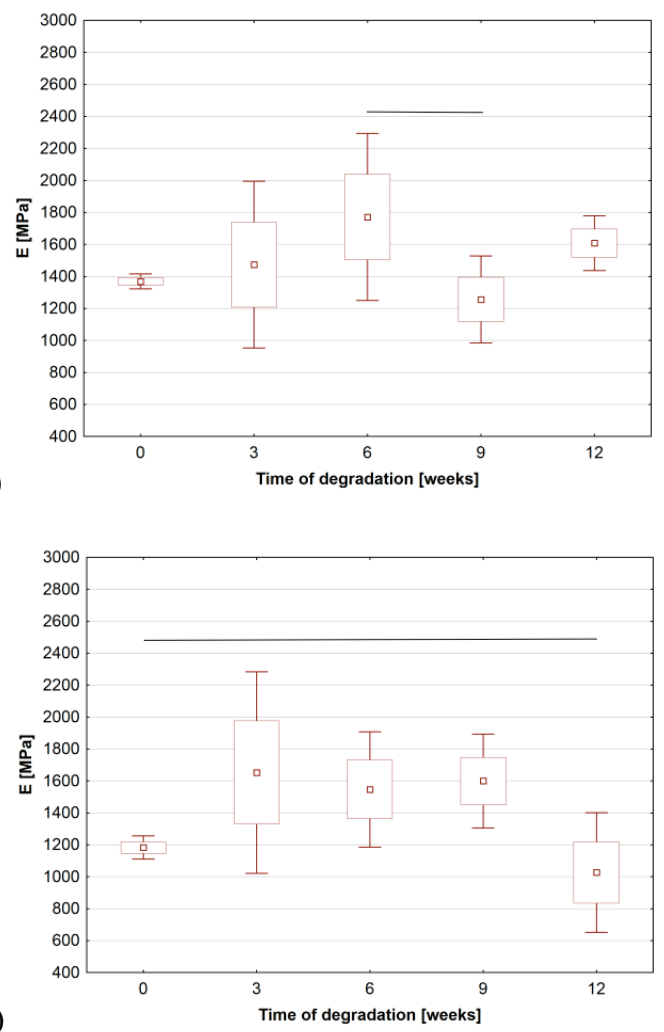

Fig. 2. Young's modulus $(E)$ and tensile strength $\left(R_{m}\right)$ after the completion of successive stages of hydrolytic degradation of PLGA specimens: (a) and (b) reference (unmodified) and modified with a $\mathrm{CO}_{2}$ laser at fluences:

(c) and (d) P1, (e) and (f) P2, and (g) and (h) P3. The data are presented as follows: mean as the central point, standard deviation as the box, and $\mathrm{min} / \mathrm{max}$ range as whiskers. The buckle indicates the statistical significance of the Friedman test for dependent groups at the $\alpha=0.05$ 
Tensile strength

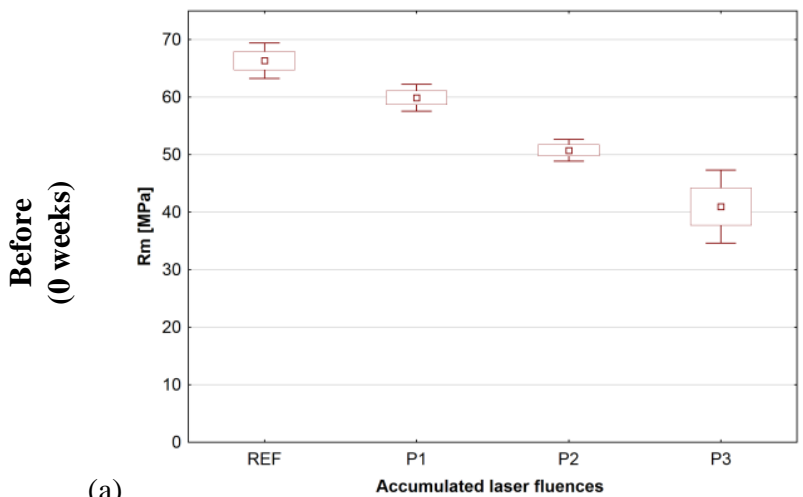

(a)

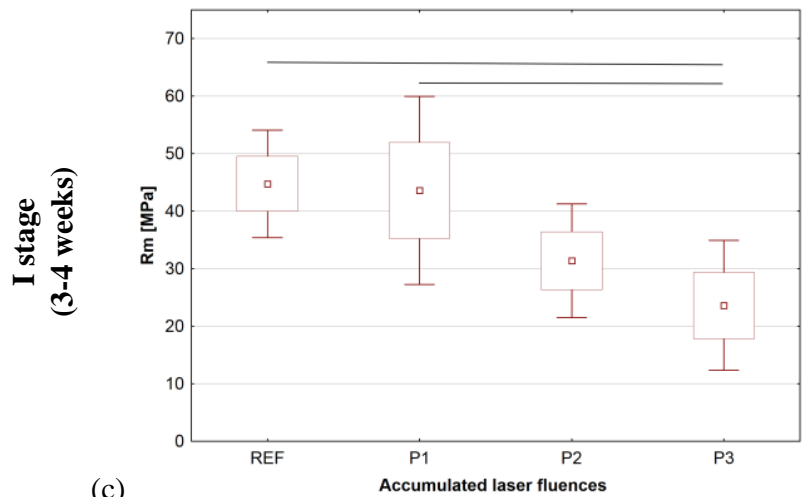

(c)
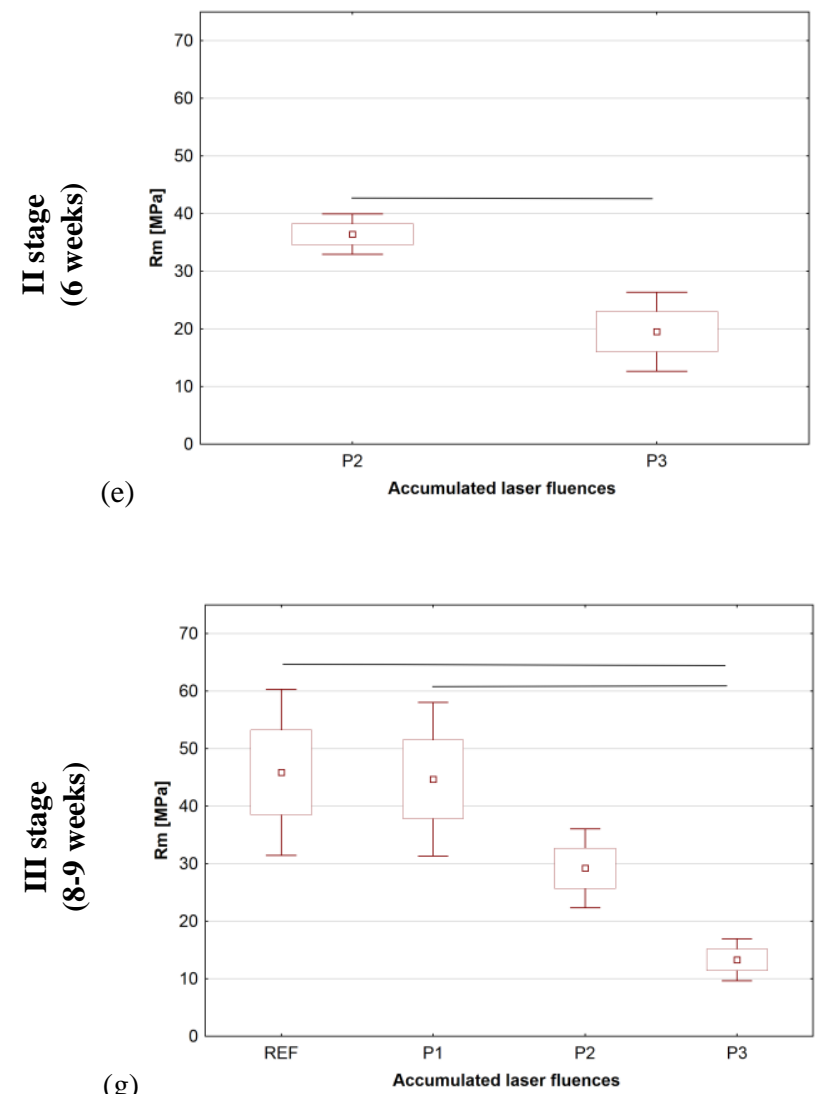

Young's modulus
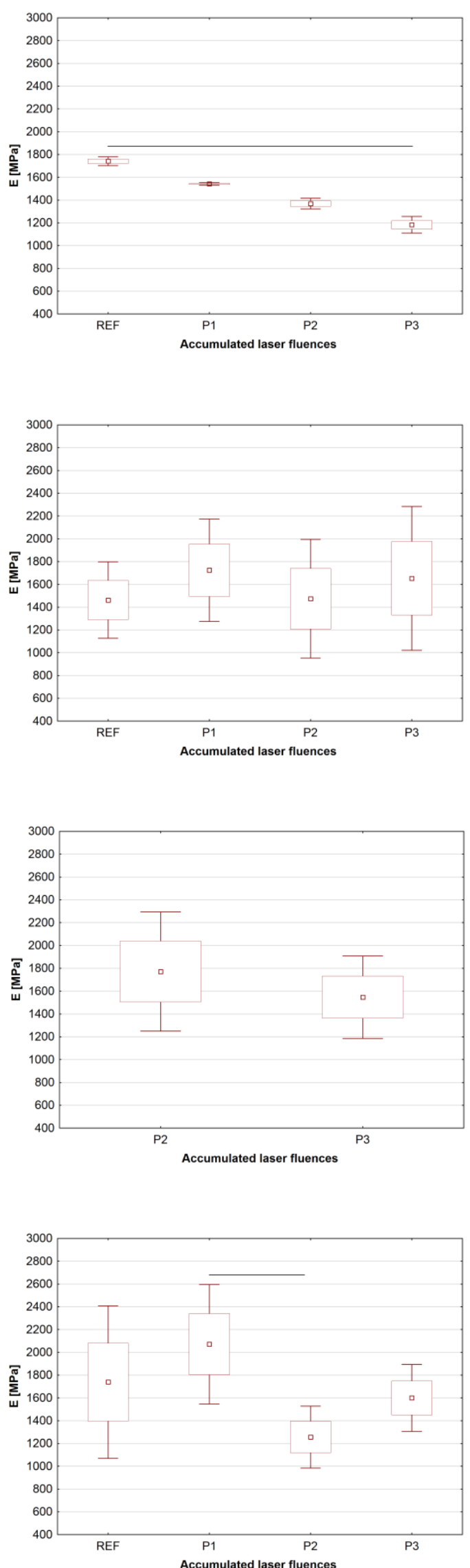

(h) 

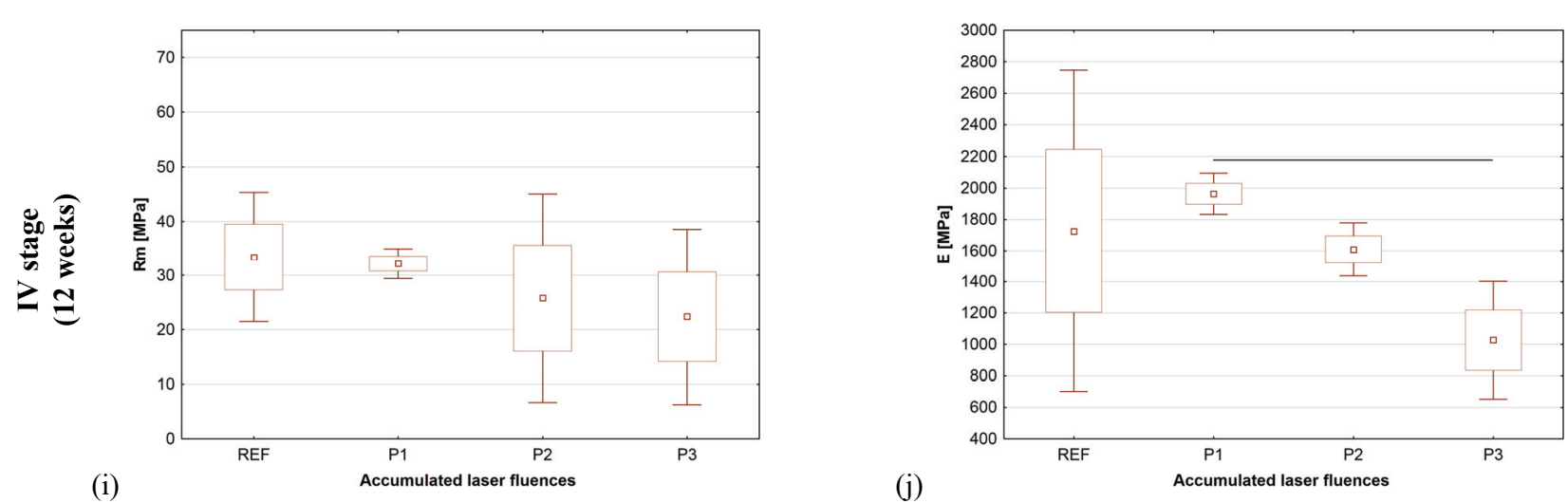

Fig. 3. Young's modulus $(E)$ and tensile strength $\left(R_{m}\right)$ of reference (unmodified) PLGA specimens and of the specimens modified by a $\mathrm{CO}_{2}$ laser with $\mathrm{P} 1, \mathrm{P} 2$, and $\mathrm{P} 3$ fluences at individual stages of hydrolytic degradation:

(a) and (b) before degradation (0 weeks), (c) and (d) after stage I of the degradation (third or fourth week),

(e) and (f) after stage II of the degradation (sixth week), (g) and (h) after stage III of the degradation (eighth or ninth week), and (i) and (j) after stage IV of the degradation (twelfth week). The data are presented as follows: mean as the central point, standard deviation as the box, and $\mathrm{min} / \mathrm{max}$ range as whiskers. The buckle indicates the statistical significance of the Kruskal-Wallis test for dependent groups at the $\alpha=0.05$

results, resulting in a large scatter and value of the standard deviation. Such a large variability of the obtained $E$ values was most likely a consequence of the progressing specimen decomposition, which did not occur uniformly throughout the group. For all modification cases and references specimens, the recorded scatter of the results was significantly lower before the degradation (Figs. 3a and 3b) than during the degradation. This confirms the heterogeneous nature of the degradation in aqueous environment of both unmodified PLGA and PLGA modified by a $\mathrm{CO}_{2}$ laser, regardless of the applied fluence.

\subsection{Surface topography}

At the first stage of the analysis, the PLGA_REF reference material was tested. Before the degradation process, the PLGA_REF specimens were character-

(a)

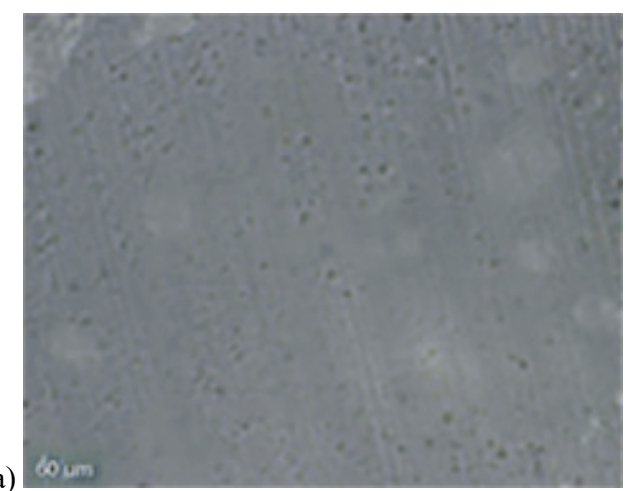

ised by a smooth, homogeneous surface with slight changes typical of the process of their manufacture (Fig. 4a). In the first stage of the degradation, the specimens became slightly tarnished, and in the successive weeks, the unevenness of the surface increased. After 12 weeks of the degradation, the PLGA_REF surface underwent visible, focal changes accompanied by a significant increase in its roughness (Fig. 4b).

The surfaces of the PLGA P1 specimens before the degradation showed a characteristic structure of parallel dark and light lines, indicating the presence of longitudinal grooves and ridges (Fig. 5a). An increase in fluence used for modification led to a dispersion of the clear pattern forming on the material surface (Figs. $5 \mathrm{~b}$ and 5c). Polymer hydrolytic degradation resulted in blurring and loss of the clear pattern of grooves and ridges (Figs. 5d, 5e, and 5f). This trend intensified with the increase in the fluence value.

(b)

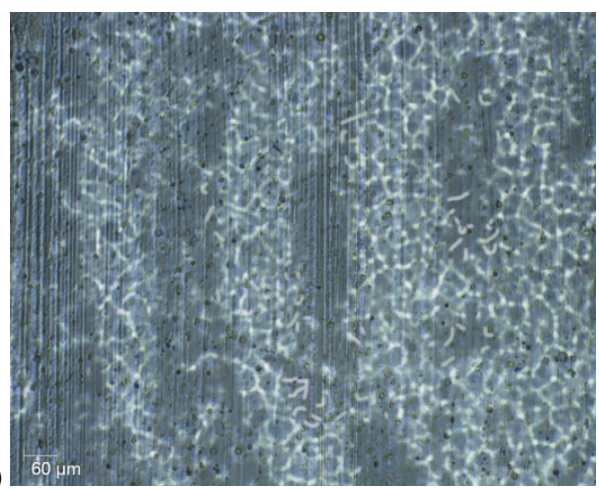

Fig. 4. Microscopic images of the surface of PLGA_REF (without laser modification):

(a) before and (b) after the end of hydrolytic degradation 
Below the ablation threshold (P1)
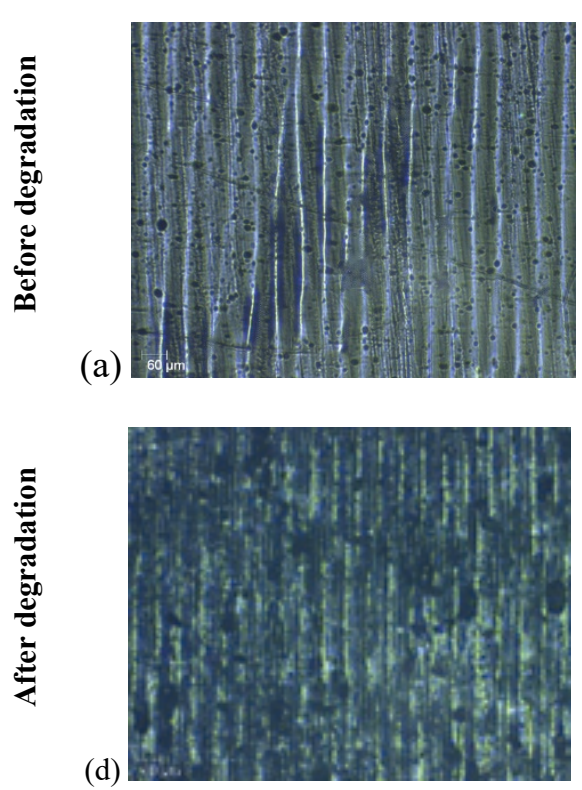

At the ablation threshold

(P2)
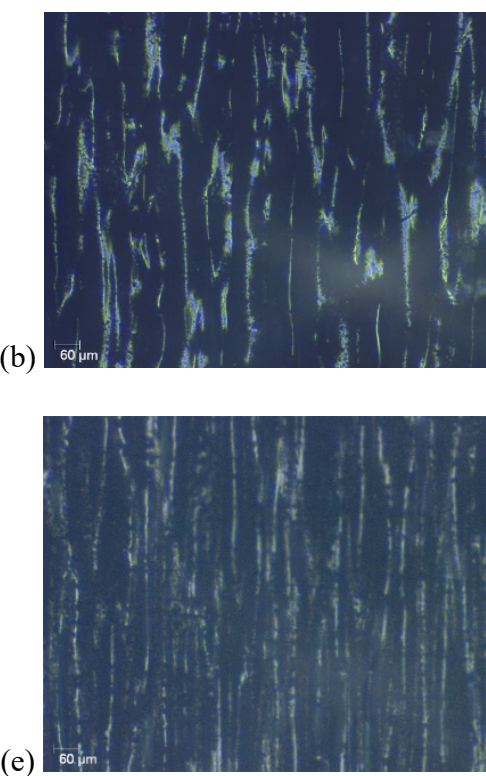

Above the ablation threshold

(P3)

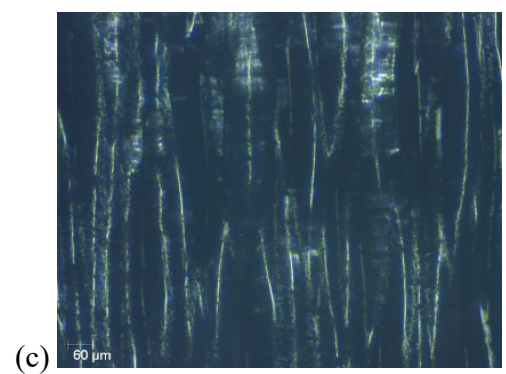

(f)

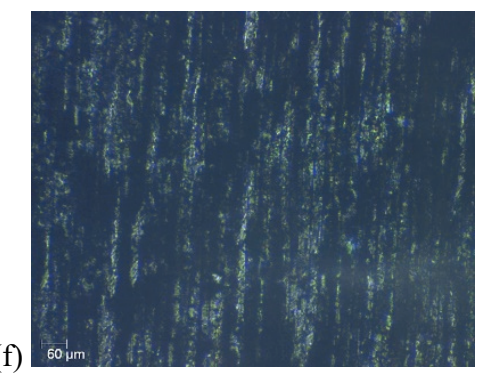

Fig. 5. Microscopic images of the surface topography of PLGA specimens modified by a $\mathrm{CO}_{2}$ laser with different fluences before and after the end of a 12-week hydrolytic degradation

\subsection{Mass and geometric dimensions of the specimens}

The geometric dimensions (width and thickness) of the specimens as well as masses of the specimens were collected in Table 3 in the form of means with standard deviation $(\bar{X} \pm S D)$.

Both the width and thickness of the PLGA reference specimens decreased at each degradation stage, relatively to the baseline measurement made before

Table 3. Geometric dimensions and masses of PLGA specimens determined during hydrolytic degradation

\begin{tabular}{|c|c|c|c|c|c|c|c|}
\hline \multirow{2}{*}{$\begin{array}{l}\text { Degradation } \\
\text { time } \\
\text { [weeks] }\end{array}$} & \multicolumn{2}{|c|}{ Thickness } & \multicolumn{2}{|c|}{ Width } & \multicolumn{3}{|c|}{ Mass } \\
\hline & $\begin{array}{c}\text { before degradation } \\
{[\mathrm{mm}]}\end{array}$ & $\begin{array}{c}\text { after degradation } \\
{[\mathrm{mm}]}\end{array}$ & $\begin{array}{l}\text { before degradation } \\
{[\mathrm{mm}]}\end{array}$ & $\begin{array}{c}\text { after degradation } \\
{[\mathrm{mm}]}\end{array}$ & $\begin{array}{c}\text { before degradation } \\
{[\mathrm{g}]}\end{array}$ & $\begin{array}{l}\text { after degradation } \\
{[\mathrm{g}]}\end{array}$ & $\begin{array}{l}\text { after } 48 \text { hours } \\
\text { of drying [g] }\end{array}$ \\
\hline \multicolumn{8}{|c|}{ Reference material (REF) } \\
\hline 0 & $0.261 \pm 0.017$ & - & $3.920 \pm 0.024$ & - & $0.237 \pm 0.029$ & - & - \\
\hline 4 & $0.339 \pm 0.051$ & $0.329 \pm 0.049$ & $4.019 \pm 0.043$ & $3.995 \pm 0.048$ & $0.229 \pm 0.034$ & $0.231 \pm 0.035$ & $0.028 \pm 0.034$ \\
\hline 8 & $0.277 \pm 0.019$ & $0.262 \pm 0.015$ & $4.033 \pm 0.034$ & $3.939 \pm 0.017$ & $0.198 \pm 0.015$ & $0.200 \pm 0.016$ & $0.198 \pm 0.016$ \\
\hline 12 & $0.305 \pm 0.024$ & $0.291 \pm 0.027$ & $3.995 \pm 0.042$ & $3.941 \pm 0.041$ & $0.213 \pm 0.021$ & $0.219 \pm 0.021$ & $0.213 \pm 0.022$ \\
\hline \multicolumn{8}{|c|}{ Below the ablation threshold (P1) } \\
\hline 0 & $0.279 \pm 0.006$ & - & $3.982 \pm 0.024$ & - & $0.215 \pm 0.023$ & - & - \\
\hline 4 & $0.260 \pm 0.028$ & $0.246 \pm 0.025$ & $3.957 \pm 0.038$ & $4.019 \pm 0.020$ & $0.191 \pm 0.025$ & $0.192 \pm 0.024$ & $0.191 \pm 0.025$ \\
\hline 8 & $0.274 \pm 0.055$ & $0.262 \pm 0.044$ & $3.963 \pm 0.029$ & $3.967 \pm 0.021$ & $0.195 \pm 0.026$ & $0.198 \pm 0.025$ & $0.195 \pm 0.026$ \\
\hline 12 & $0.286 \pm 0.024$ & $0.271 \pm 0.023$ & $3.971 \pm 0.026$ & $4.055 \pm 0.051$ & $0.208 \pm 0.011$ & $0.213 \pm 0.012$ & $0.212 \pm 0.017$ \\
\hline \multicolumn{8}{|c|}{ At the ablation threshold (P2) } \\
\hline 0 & $0.357 \pm 0.007$ & - & $4.013 \pm 0.033$ & - & $0.241 \pm 0.017$ & - & - \\
\hline 3 & $0.302 \pm 0.029$ & $0.296 \pm 0.027$ & $3.907 \pm 0.007$ & $3.910 \pm 0.040$ & $0.222 \pm 0.020$ & $0.231 \pm 0.018$ & $0.207 \pm 0.022$ \\
\hline 6 & $0.290 \pm 0.027$ & $0.286 \pm 0.021$ & $3.927 \pm 0.031$ & $3.932 \pm 0.041$ & $0.210 \pm 0.013$ & $0.211 \pm 0.013$ & $0.209 \pm 0.014$ \\
\hline 9 & $0.289 \pm 0.030$ & $0.288 \pm 0.030$ & $3.899 \pm 0.050$ & $3.914 \pm 0.051$ & $0.215 \pm 0.020$ & $0.218 \pm 0.021$ & $0.214 \pm 0.021$ \\
\hline 12 & $0.280 \pm 0.033$ & $0.238 \pm 0.030$ & $3.883 \pm 0.039$ & $3.927 \pm 0.084$ & $0.201 \pm 0.021$ & $0.223 \pm 0.041$ & $0.199 \pm 0.022$ \\
\hline \multicolumn{8}{|c|}{ Above the ablation threshold (P3) } \\
\hline 0 & $0.256 \pm 0.034$ & - & $3.945 \pm 0.076$ & - & $0.221 \pm 0.031$ & - & - \\
\hline 3 & $0.245 \pm 0.022$ & $0.236 \pm 0.026$ & $3.701 \pm 0.080$ & $3.746 \pm 0.092$ & $0.206 \pm 0.018$ & $0.211 \pm 0.017$ & $0.204 \pm 0.016$ \\
\hline 6 & $0.217 \pm 0.013$ & $0.215 \pm 0.013$ & $3.647 \pm 0.097$ & $3.649 \pm 0.066$ & $0.183 \pm 0.016$ & $0.189 \pm 0.018$ & $0.182 \pm 0.016$ \\
\hline 9 & $0.233 \pm 0.015$ & $0.215 \pm 0.035$ & $3.776 \pm 0.060$ & $3.710 \pm 0.020$ & $0.191 \pm 0.013$ & $0.191 \pm 0.012$ & $0.188 \pm 0.013$ \\
\hline 12 & $0.250 \pm 0.017$ & $0.181 \pm 0.030$ & $3.696 \pm 0.079$ & $3.657 \pm 0.103$ & $0.195 \pm 0.023$ & $0.195 \pm 0.025$ & $0.190 \pm 0.024$ \\
\hline
\end{tabular}


the start of the degradation. Modifications of PLGA with P1 and P2 fluences at all degradation stages and with the P3 fluence up to and including the sixth week caused an increase in the mean width and a decrease in the thickness of the specimens. In the 9th and 12th week of incubation of PLGA_P3, there was a visible reduction of the geometric dimensions of the specimens. The masses of these specimens before the start of the degradation and immediately after its completion did not change in contrast to the recorded mass increases in all other cases. The increase in the specimen masses during the incubation was due to the phenomenon of water absorption by the material. After 48 hours of air drying, the specimen masses were slightly lower than the specimen masses measured immediately after each completed incubation stage. At each degradation stage, the masses of PLGA_P2 and PLGA_P3 after drying were lower than before the start of the degradation, whereas no differences were found for PLGA_REF and PLGA_P1.

\section{4. $\mathrm{pH}$ of the degradation environment}

The $\mathrm{pH}$ values of the incubation solutions were measured every 2 weeks. The incubation solutions of

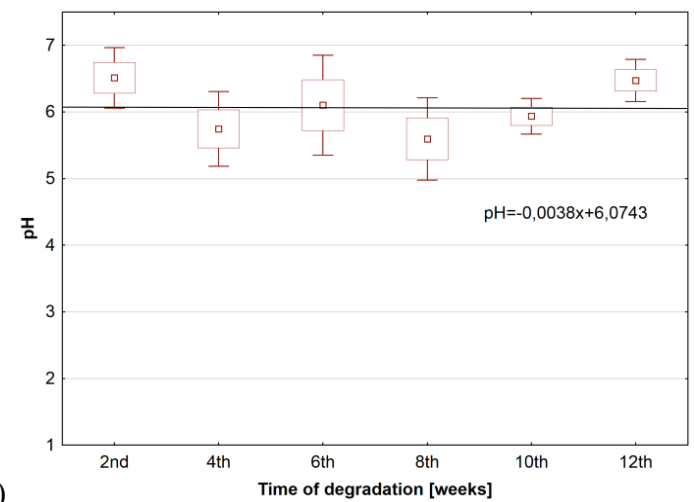

(a)

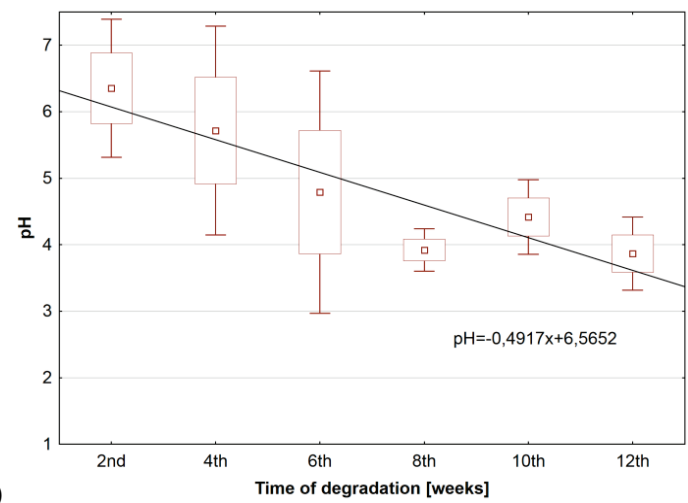

both the reference material and the PLGA specimens subjected to all three cases of modification showed a gradual decrease in the $\mathrm{pH}$ values with the degradation time (Fig. 6), as evidenced by the negative value of the $a$ parameter of the linear function approximating the mean $\mathrm{pH}$ values in the successive weeks of the degradation. The value of the $a$ parameter for PLGA_REF (Fig. 6a) was close to zero, proving that the degradation process proceeded at a similar rate throughout the test cycle. In the case of incubation of PLGA_P1 (Fig. 6b) and PLGA_P2 (Fig. 6c) specimens, the $\mathrm{pH}$ of the solution gradually decreased in the successive weeks of the degradation, demonstrating the progressive degradation of the material. The dynamics of this process increased with an increase in the value of the applied fluence (Figs. 6b and 6c), as evidenced by the decreasing value of the $a$ parameter. In the case of PLGA modifications with P3 fluence (Fig. 6d), the dynamics of the $\mathrm{pH}$ changes was comparable to those observed for PLGA_P1, but a rapid decrease in the $\mathrm{pH}$ level occurred already in the second week of incubation. This means that the degradation process of PLGA_P3 had a different course - the process was initiated very early after the start of the degradation, and then its course stabilised.

(b)

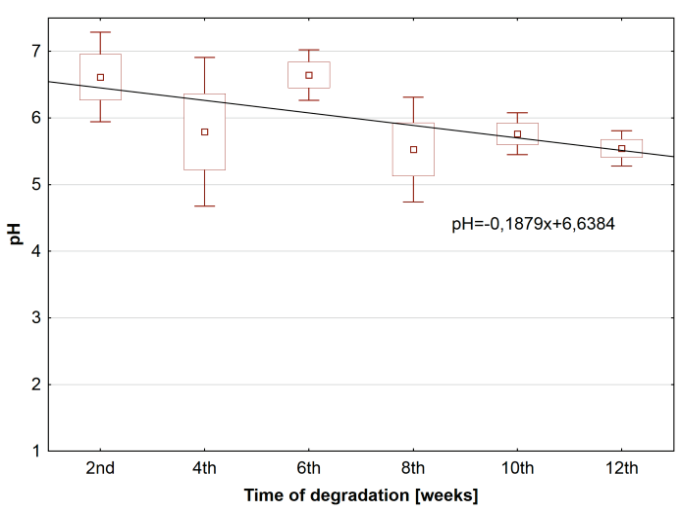

(d)

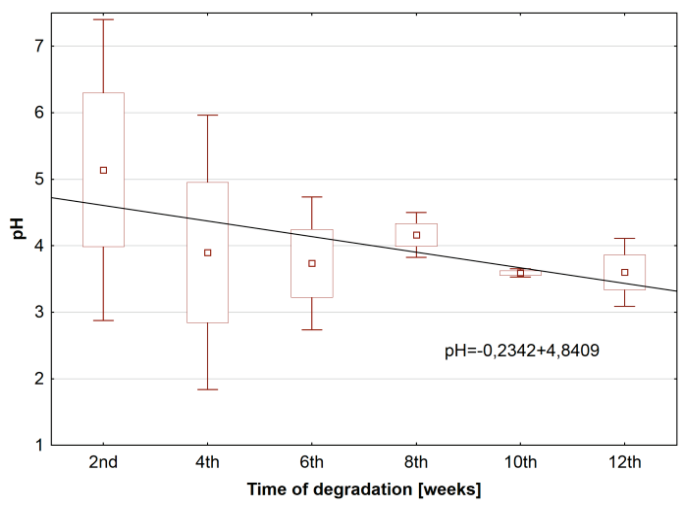

Fig. 6. $\mathrm{pH}$ in successive weeks of the degradation for (a) PLGA reference specimens and PLGA specimens modified by a $\mathrm{CO}_{2}$ laser with the fluences of (b) P1, (c) P2, and (d) P3 


\section{Discussion}

Bioresorbable polymers have been known for many years as a biomaterial with enormous application potential, including in biomedicine. Of particular interest is the use of aliphatic polymers for the production of tissue scaffolds and bioresorbable implants with small geometric dimensions. One advantage of the materials from this group is the broad ability to shape their composition and topography in order to obtain the intended functionality. This study presents research aimed at assessing the effects of surface modifications of the PLGA copolymer by a $\mathrm{CO}_{2}$ laser with different process parameters (Table 1) on the mechanical properties of the specimens subjected to hydrolytic degradation (Figs. 1, 2, and 3). The research analysed three cases of PLGA surface modification: (i) without ablation (P1), (ii) by ablation with reduced thermal effects (P2), and (iii) by ablation with the accompanying process of thermal degradation (P3), correlated with the values of accumulated laser fluencies. After irradiation, the specimens were incubated in distilled water for 12 weeks in accordance with the calendar (Table 2). In the successive stages of incubation, the analysis covered the mechanical properties and geometric dimensions and masses of the reference specimens (untreated by laser) and the specimens modified by a $\mathrm{CO}_{2}$ laser with three different accumulated laser fluences as well as the $\mathrm{pH}$ value of the incubation environment.

In the case of the compared specimens, the degradation process proceeded with different dynamics. During the incubation, constant and reproducible conditions were ensured for diffusion of degradation products into the incubation environment by changing the solution for each specimen every 2 weeks. This allowed to assess the rate of release of degradation products. In water, PLGA undergoes biodegradation through hydrolysis of ester bonds [6], as a result of which acidic products of hydrolysis are released into the incubation environment, thus reducing $\mathrm{pH}$ [23]. By analysing the changes in the $\mathrm{pH}$ of the incubation environment, it was concluded that in the case of the reference material not subjected to laser modification, the degradation process proceeded evenly throughout the observation period (Fig. 6a). This indicates a constant rate of release of the degradation products. In the case of modified specimens, the degradation rate was much higher than for the reference specimens, and changes in the $\mathrm{pH}$ value of the incubation solution suggest a different nature of the degradation for modifications of P1 and P2 (Figs. 6b and 6c) compared to
P3 (Fig. 6d). Modification of PLGA as a result of ablation and thermal degradation (P3) caused not only evaporation of the material from the surface but also its melting [2]. The $\mathrm{pH}$ of the solution in which PLGA_P3 specimens were incubated dropped sharply after two weeks, and then the process continued at a lower rate. The observed effects may be due to the presence on the specimen surface of a highly degraded material, weakly bound to the rest of the specimen [25], which caused faster release of degradation products, resulting in increased acidity of the incubation environment.

The stress-strain characteristics of the PLGA specimens determined on the basis of a uniaxial tensile test indicate that the applied laser treatment changed the material model. In the case of reference specimens and PLGA specimens irradiated with a $\mathrm{CO}_{2}$ laser, there was a gradual transition from ductile failure characteristic of untreated PLGA to brittle failure of laser-modified materials [20o-_ENREF_19]. Regardless of the considered case of surface modification, the range of plastic deformations and strain softening decreased due to deformation (Fig. 1). As the degradation time increased, the plastic region and strain softening became smaller; after 12 weeks of the degradation, brittle failure of PLGA_REF and all types of surface modification (P1, P2 and P3) were observed. There were no observed strain hardening for any cases of modification or at any degradation stage. That would be characteristic of polymers composed of an entangled network [11]. This was due to a strong processing of the surface layer of the material as a result of laser irradiation, which was accompanied by an increase in the degree of crystallinity proportional to the fluence value [14]. Typically, higher crystallinity of aliphatic polyesters results in a brittle material without ductile deformation [21].

Young's modulus and tensile strength were determined in accordance with the PN-EN ISO 527-3:1998 standard [12] for all types of specimens at all stages of the degradation. The determined values of tensile strength and Young's modulus of the reference PLGA before degradation were, respectively, $66.4 \pm 1.6 \mathrm{MPa}$ and $1,740.8 \pm 19.4 \mathrm{MPa}$, corresponding with the literature data [1], [27]. The very process of irradiation of PLGA specimens caused a reduction of the tensile strength and Young's modulus values, which was described in detail in a previous study by the authors of this article [14]. Incubation of unmodified PLGA in a water bath decreased the mean values of tensile strength (Fig. 2a) without significantly changing the mean values of Young's modulus (Fig. 2b). Bartkowiak-Jowsa et al. [3] in the same observation period 
(3 months) for pure PLGA noted an abrupt reduction in tensile strength after the first month of incubation, followed by a plateau, and then a gradual but slight reduction in Young's modulus. The mechanical properties of PLGA specimens modified by a $\mathrm{CO}_{2}$ laser during the hydrolytic degradation test also changed. Tensile strength decreased with degradation time, regardless of the case of surface modification. After the first stage of the degradation (third or fourth week), the Rm values fell rapidly (Fig. 2), and then the dynamics of changes decreased or there was a plateau as in the study by Bartkowiak-Jowsa et al. [3]. The percentage reduction in tensile strength after the first stage of the degradation of modified PLGA specimens amounted to $\approx 25 \%, \approx 38 \%$, and $\approx 42 \%$ for $\mathrm{P} 1, \mathrm{P} 2$, and $\mathrm{P} 3$, respectively. After 12 weeks of incubation, the percentage decrease in the tensile strength values for all compared groups of specimens was similar and amounted to $\approx 46 \%, \approx 49 \%$, and $\approx 45 \%$ for $\mathrm{P} 1, \mathrm{P} 2$, and $\mathrm{P} 3$, respectively. The mean value of Young's modulus determined for the modified PLGA specimens in the first stages of the degradation increased insignificantly, which may be the result not of the degradation process itself, but of the recorded heterogeneous changes in the values of geometric dimensions of the specimens (Table 3), and thus the values of the crosssectional area (data are not presented here) after incubation. These changes occurred as a result of water absorption. It is also worth emphasising that the results obtained in all stages of the degradation were characterized by significant variability. For all modification cases and references specimens, the recorded scatter of the results was significantly lower before the degradation (Figs. 3a and 3b) than during the degradation. This was due to the progressing specimen decomposition, which was heterogeneous for both unmodified PLGA and specimens modified by a $\mathrm{CO}_{2}$ laser regardless of the applied fluence, as a result of the degradation in aqueous environment. The reasons for this phenomenon can be found in the weakened links between mers, which are found in aliphatic polyester films, including PLGA, provided that the oligomers [23] are present in the material structure. The presence of oligomers in the examined PLGA specimens was not confirmed experimentally, but both the process of specimen irradiation and incubation in an aqueous solution cause the breakage of chemical bonds in the macromolecule and the formation of shorter fragments with a much lower molecular weight, i.e., oligomers [2], [28].

The results presented in this study concern the assessment of changes in the mechanical properties of PLGA under the influence of hydrolytic degradation.
The degradation time of PLGA depends on the ratio of lactide to glycolide in the polymer chain and is 4-8 weeks for PLGA in the 50:50 lactide to glycolide ratio, 16-20 weeks for the 75:25 ratio, and 20-24 weeks for the $85: 15$ ratio [16]. PLGA has found application in the production of regenerated bone, scaffolds, drug carriers, and sutures, where its short degradation time is an advantage. In vitro, the implants are subjected to not only the degrading action of the incubation environment but also various mechanical, static or dynamic loads, including highly cyclic loads resulting from normal life functions. As evidenced by Guo et al. [9], polymers undergoing hydrolytic degradation while being subjected to loading show more intense changes. This aspect should be considered when designing bioresorbable medical devices made of PLGA, especially after subjecting the material to surface modification, which intensifies the process of material degradation.

An unquestionable advantage of bioresorbable polymers is the ability to obtain a gradual reduction of load-bearing properties of an implant through material degradation in order to ensure appropriate biomechanical conditions for the reconstruction and remodelling of the tissue structure. On the other hand, the implant should maintain its geometry for a predetermined period of time necessary for effective treatment and regeneration of tissue. The results of the conducted research showed that geometric dimensions of the PLGA reference specimens decreased at each stage of the degradation. On the other hand, geometric dimensions of PLGA modified with P1 and P2 fluences did not change significantly during the 12-week incubation in distilled water. Moreover, the masses of the PLGA_P1 and PLGA_P2 specimens after a 48-hour drying period did not change at any degradation stage. Only in the 9th and 12th week of incubation for the case of PLGA_P3 modification, there was a significant reduction in the geometric dimensions and masses. The increase in the mass observed for all the specimens immediately after incubation was due to the phenomenon of water absorption. After 48-hour drying at room temperature, the absorbed water evaporated. This phenomenon is typical of amorphous materials [8].

Observations of polymer surface topographies showed the presence of characteristic structures that were the result of the technology of irradiating PLGA surfaces with a $\mathrm{CO}_{2}$ laser. Modifications of PLGA without ablation resulted in a clear pattern of parallel and equidistant grooves and ridges repeating over a distance of 40-60 $\mu \mathrm{m}$. Irradiation of PLGA with P2 and P3 fluences also resulted in the above repetitive pattern, however, it was characterized by a lower degree 
of ordering, with less clearly visible boundaries between grooves and ridges. The performed hydrolytic degradation had a slight influence on the surface topography of the analysed materials. After the end of the degradation process, there were small visible changes, causing in microscopic evaluation a blurring of the boundaries between the structures originally formed on the surface. This type of surface structuring can be used for the purpose of its functionalisation or structuralization, especially considering the fact that in the analysed PLGA the induced surface topography was stable and underwent only slight changes in the 12-week incubation period. On the other hand, in the course of polymer degradation, there was a gradual tarnishing of the surfaces of the specimens. It was associated with a progressive hydrolysis of the polyester bonds, which resulted in the formation of shorter chains. Short chains of higher mobility form ordered structures, thereby changing the optical properties of the material confirmed their crystallization [24].

The analyses presented in this study contribute scientifically to research studies on the production of implants from bioresorbable polymers with the use laser modifications [2], [3], [14], [25]. The purpose of the comprehensive assessment of the effects of implant production processes and technologies is to maintain the characteristics of the material or their optimal modification in a strictly intended manner.

\section{Limitations}

The main limitation of this study is the number of specimens: the size of each group was 5 for each modification case in successive degradation stages. In order to compensate for the low number of specimens in the groups, nonparametric statistical tests were used to define the significance of the differences in the obtained results. At the same time, the results are presented as means with standard deviations, which is a deliberate procedure allowing easier comparison of the obtained data with the state-of-the-art knowledge. Due to the lower power of nonparametric tests, which translates into a lower accuracy of identification of statistically significant differences, the analysis also covered the trends and directions of changes.

The second limitation of this study is the duration of the degradation, set at 12 weeks due to expected increased susceptibility of the specimens subjected to surface modification by a $\mathrm{CO}_{2}$ laser to the degradation in aqueous solution. The 12-week incubation for PLGA_REF and PLGA_P1 specimens enabled the authors to determine the trend of changes in mechani- cal parameters, while the nature of changes in the case of geometric dimensions or masses was not clearly defined. A longer incubation period would allow to identify the time necessary to disintegrate the specimens, which could be defined by the specimens' geometrical dimensions and their masses.

\section{Acknowledgements}

This study was carried out with financial support from the National Science Centre on the basis of the decision number DEC-2013/09/B/ST8/02423.

\section{References}

[1] Al-Sukhun J., Kontio R., Lindqvist C., Bioresorbable poly-L/DL-lactide $[P(L / D L) L A$ 70/30] plates are reliable for repairing of large inferior orbital wall bony defects: A pilot study, J. Oral. Maxil. Surg., 2006, 64, 47-55.

[2] AntończaK A.J., StęPak B.D., Szustakiewicz K., Wóıcik M.R., ABRAMSKi K.M., Degradation of poly(l-lactide) under $\mathrm{CO}_{2}$ laser treatment above the ablation threshold, Polym. Degrad. Stabil., 2014, 109, 97-105.

[3] Bartkowiak-Jowsa M., BęDziński R., KozŁowska A., FilipiaK J., Pezowicz C., Mechanical, rheological, fatigue, and degradation behavior of PLLA, PGLA and PDGLA as materials for vascular implants, Meccanica, 2013, 48, 721-731.

[4] Bhatla A., YaO Y.L., Effect of laser surface modification on the crystallinity of poly(l-lactic acid), J. Manuf. Sci. Eng., 2009, 131, 51004-1-51004-11.

[5] Carrasco F., Pagès P., Gámez-Pérez J., Santana O.O., MASPOCH M.L., Processing of poly(lactic acid): Characterization of chemical structure, thermal stability and mechanical properties, Polym. Degrad. Stabil., 2010, 95, 116-125.

[6] Elmowafy E.M., Tiboni M., Soliman M.E., Biocompatibility, biodegradation and biomedical applications of poly(lactic acid)/poly(lactic-co-glycolic acid) micro and nanoparticles, J. Pharm. Investig., 2019, 49, 347-380.

[7] Farah S., Anderson D.G., Langer R., Physical and mechanical properties of PLA, and their functions in widespread applications - A comprehensive review, Adv. Drug. Deliv. Rev., 2016, 107, 367-392.

[8] FRANK D.S., MATZGER A.J., Effect of polymer hydrophobicity on the stability of amorphous solid dispersions and supersaturated solutions of a hydrophobic pharmaceutical, Mol. Pharm., 2019, 16, 682-688.

[9] Guo M., Chu Z., Yao J., Feng W., Wang Y., Wang L., Fan Y., The effects of tensile stress on degradation of biodegradable PLGA membranes: A quantitative study, Polym. Degrad. Stabil., 2016, 124, 95-100.

[10] Hines D.J., KaPLAN D.L., Poly(lactic-co-glycolic) acid-controlled-release systems: Experimental and modeling insights, Crit. Rev. Ther. Drug. Carrier Syst., 2013, 30, 257-276.

[11] HoY R.S., Why is understanding glassy polymer mechanics so difficult?, J. Polym. Sci. Pol. Phys., 2011, 49, 979-984.

[12] InTERNATIONAL, ORGANIZATION FOR STANDARDIZATION ISO 527-1:2012, Plastics - Determination of tensile properties - Part 1: General principles, Geneva 2012. 
[13] Jia W., Luo Y., Yu J., Liu B., Hu M., Chai L., Wang C., Effects of high-repetition-rate femtosecond laser micromachining on the physical and chemical properties of polylactide (PLA), Opt. Express, 2015, 23, 26932-26939.

[14] Kobielarz M., Gazińska M., TomaniK M., StęPak B., Szustakiewicz K., Filipiak J., AntońcZak A., Pezowicz C., Physicochemical and mechanical properties of $\mathrm{CO}_{2}$ laser-modified biodegradable polymers for medical applications, Polym. Degrad. Stabil., 2019, 165, 182-195.

[15] Laput O., Vasenina I., Salvadori M.C., Savkin K., ZuZa D., KURZINA I., Low-temperature plasma treatment of polylactic acid and PLA/HA composite material, J. Mater. Sci., 2019, 54, 11726-11738.

[16] Lieger O., Schaller B., ZiX J., Kellner F., IIZUKA T., Repair of orbital floor fractures using bioresorbable poly-L/DL-lactide plates, Arch. Facial. Plast. Surg., 2010, 12, 399-404.

[17] Middleton J.C., Tipton A.J., Synthetic biodegradable polymers as orthopedic devices, Biomaterials, 2000, 21, 2335-2346.

[18] MoraczewsKi K., RytlewsKi P., MAlinOwSKI R., ZENKIEWICZ M., Comparison of some effects of modification of a polylactide surface layer by chemical, plasma, and laser methods, Appl. Surf. Sci., 2015, 346, 11-17.

[19] Nikolskaya E., Sokol, M., Faustova M., Zhunina O., Mollaev M., Yabbarov N., Tereshchenko O., Popov R., SEVERIN E., The comparative study of influence of lactic and glycolic acids copolymers type on properties of daunorubicin loaded nanoparticles and drug release, Acta Bioeng. Biomech., 2018, 20 (1), 65-77.

[20] Ozdemir M., SAdikoglu H., A new and emerging technology: Laser-induced surface modification of polymers, Trends Food Sci. Tech., 1998, 9, 159-167.

[21] Renouf-Glauser A.C., Rose J., Farrar D.F., CAmEron R.E., The effect of crystallinity on the deformation mechanism and bulk mechanical properties of PLLA, Biomaterials, 2005, 26, 5771-5782.

[22] Rytlewski P., Mróz W., Zenkiewicz M., Czwartos J., BUDNER B., Laser induced surface modification of polylactide, J. Mater. Process Tech., 2012, 212, 1700-1704.

[23] Schliecker G., Schmidt C., Fuchs S., Wombacher R., KISSEL T., Hydrolytic degradation of poly(lactide-co-glycolide) films: Effect of oligomers on degradation rate and crystallinity, Int. J. Pharm., 2003, 266, 39-49.

[24] Sikorska W., Musioł M., Rydz J., Zięba M., Rychter P., LEWICKA K., ŠIšKova A., MosnÁČKOVÁ K., KowAlCZUK M., ADAMUS G., Prediction studies of environment-friendly biodegradable polymeric packaging based on PLA. Influence of specimens' thickness on the hydrolytic degradation profile, Waste Manage, 2018, 78, 938-947.

[25] StepaK B., AntończaK A.J., BartKowiak-Jowsa M., FilipiaK J., Pezowicz C., Abramski K.M., Fabrication of a polymer-based biodegradable stent using a $\mathrm{CO}_{2}$ laser, Arch. Civ. Mech. Eng., 2014, 14, 317-326.

[26] ŚCIGAla K., BĘDZiŃSKI R., FiliPIAK J., Chlebus E., DyBAŁa B., Application of generative technologies in the design of reduced stiffness stems of hip joint endoprosthesis, Arch. Civ. Mech. Eng., 2011, 11, 753-767.

[27] VERT M., Bioresorbable polymers for temporary therapeutic applications, Macromol. Mater. Eng., 1989, 166, 155-168.

[28] Wang L., Zhang Z., Chen H., Zhang S., Xiong C., Preparation and characterization of biodegradable thermoplastic elastomers (PLCA/PLGA blends), J. Polym. Res., 2010, 17, 77-82.

[29] Wang S., Cui W., Bei J., Bulk and surface modifications of polylactide, Anal. Bioanal. Chem., 2005, 381, 547-556.

[30] Waugh D.G., Lawrence J., Walton C.D., Zakaria R.B., On the effects of using $\mathrm{CO}_{2}$ and $\mathrm{F}_{2}$ lasers to modify the wettability of a polymeric biomaterial, Opt. Laser Technol., 2010, 42, 347-356. 